\title{
Anacetrapib: potential for the prevention and treatment of coronary heart disease
}

This article was published in the following Dove Press journal:

Research Reports in Clinical Cardiology

3 April 2013

Number of times this article has been viewed

\author{
Dylan L Steen' \\ Amit $\mathrm{V}$ Khera ${ }^{2}$ \\ Christopher P Cannon' \\ 'TIMI Study Group, Cardiovascular \\ Division, ${ }^{2}$ Department of Medicine, \\ Brigham and Women's Hospital, \\ Boston, MA, USA
}

\begin{abstract}
Despite major advances in cardiovascular care in recent decades, atherosclerotic cardiovascular disease remains the leading cause of morbidity and mortality worldwide. Statins have been shown to reduce cardiovascular events by $25 \%-40 \%$ in a dose-dependent fashion; yet additional therapies are needed to reduce vascular disease progression and acute thrombotic events. In addition to low-density lipoprotein cholesterol (LDL-C) reduction, other lipid risk factors, such as low high-density lipoprotein cholesterol (HDL-C), have created interest as therapeutic targets to lower cardiovascular risk. However, the absence of compelling data for incremental benefit of non-LDL-centric therapies in the statin era has limited their clinical use. A novel class of compounds, cholesteryl ester transfer protein (CETP) inhibitors, has demonstrated many potentially beneficial lipid-modifying effects. While in vitro and animal data for CETP inhibition have been encouraging, the initial enthusiasm for the class has been tempered by the failure of two CETP inhibitors (torcetrapib and dalcetrapib) in Phase III trials to reduce cardiovascular outcomes. Anacetrapib, a compound that causes near-complete CETP inhibition, has among its effects, robust reductions in LDL-C and lipoprotein(a) as well as dramatic increases in HDL-C. The ability of anacetrapib to reduce coronary disease events is being tested in the Randomized EValuation of the Effects of Anacetrapib Through Lipid-modification (REVEAL) trial (NCT01252953).
\end{abstract}

Keywords: anacetrapib, cholesteryl ester transfer protein, cholesteryl ester transfer protein inhibitor, atherosclerosis

\section{Introduction}

Significant progress has been made in the primary and secondary prevention of adverse cardiovascular outcomes, including myocardial infarction. Reduction in low-density lipoprotein cholesterol (LDL-C) levels, specifically with statin-based therapy, has been among the most important treatments to reduce atherosclerotic events. ${ }^{1}$ Despite therapeutic advances that have included intensive statin therapy, randomized trials have demonstrated that after an acute coronary syndrome approximately $20 \%-25 \%$ of patients will experience death or a major cardiovascular (CV) event in the next 24 months despite optimal medical therapy. ${ }^{2}$ Many investigators have proposed guideline changes, advocating earlier and more aggressive LDL-C lowering to reduce the lifetime risk of $\mathrm{CV}$ events. ${ }^{3}$ For many patients, this direction will require new, safe, LDL-C-lowering agents for those who cannot tolerate statins or meet LDL-C goals on statin monotherapy. In this regard, novel agents that further reduce LDL-C have been sought.

In addition to LDL-C reduction, modification of other known $\mathrm{CV}$ risk factors, such as high-density lipoprotein cholesterol (HDL-C) and lipoprotein(a) (Lp[a]) may be important therapeutic targets. The Framingham Heart Study first demonstrated a powerful inverse relationship between HDL-C and the risk of cardiovascular events in the 1970s. ${ }^{4}$ Since 
this initial finding, numerous experimental and observational studies have suggested a protective role for HDL against atherosclerosis, termed the "HDL hypothesis." The intimate association of HDL with proatherosclerotic conditions, the heterogeneity in its compositional and functional subclasses, and its many proposed antiatherothrombotic functions have, however, made it difficult to disentangle whether HDL (or some constituent of HDL) plays a direct protective role or whether it primarily serves as a biomarker of a proatherothrombotic state. ${ }^{5}$ Disappointingly, niacin, which augments HDL-C by $20 \%-25 \%$, recently failed to lower atherosclerotic events in both the Atherothrombosis Intervention in Metabolic Syndrome with Low HDL/High Triglycerides and Impact on Global Health Outcomes (AIM-HIGH) ${ }^{6}$ and Treatment of HDL to Reduce the Incidence of Vascular Events (HPS2-THRIVE) trials. ${ }^{7,8}$ It is possible that HDL-C augmentation by another mechanism or to higher levels might be beneficial. In contrast, Lp(a) lowering has not yet been evaluated in randomized controlled trials, but observational and genetic (including Mendelian randomization) analyses have demonstrated an independent association of increased Lp(a) levels with increased CV events, suggesting Lp(a) lowering may confer benefit. ${ }^{9}$

In this light, cholesteryl ester transfer protein (CETP), a central modulator of human lipid metabolism, has become an exciting new therapeutic target. Near-complete CETP inhibition results in significant LDL-C reduction as well as other lipid effects, including $\mathrm{Lp}$ (a) reduction and dramatic HDL-C elevation. Preclinical evidence from animal models naturally expressing this protein has demonstrated CETP inhibition reduces atherosclerosis, providing encouragement to target CETP in order to treat human disease. The surprising failure of the first two CETP inhibitors (torcetrapib and dalcetrapib) in Phase III outcomes trials has somewhat tempered this initial excitement and forced a re-evaluation of the complex effects of CETP inhibition on lipid metabolism and vascular biology. This review will focus on the potential of anacetrapib, a novel CETP inhibitor, to reduce coronary heart disease, largely based on insights from the torcetrapib and dalcetrapib experiences. Anacetrapib results in near-complete CETP inhibition with more pronounced lipid effects than its predecessors and is currently in a Phase III study for secondary prevention of coronary events. If successful it is likely that anacetrapib will also be considered for statin-intolerant patients and for primary prevention in patients who require LDL-C lowering beyond statin monotherapy.

\section{CETP as a target}

CETP originally was identified as a potential target for pharmacologic inhibition when a Japanese family was described with homozygous CETP splicing defects resulting in complete CETP deficiency. ${ }^{10}$ In homozygotes, LDL-C was approximately $80 \mathrm{mg} / \mathrm{dL}$ and HDL-C was approximately $160 \mathrm{mg} / \mathrm{dL}$, with more moderate changes in heterozygotes. ${ }^{11}$ The underlying mutation, intron 14 splice donor site $+1 \mathrm{G}>\mathrm{A}$ (Int14+1A) (allele frequency, $0.8 \%$ in the Japanese population), has since been well characterized. Since this first report it has been found that about half of Japanese hyperalphalipoproteinemia (HALP) is caused by CETP gene mutations, including the mutation of D442G (allele frequency, 3.4\% in the Japanese population). The homozygote of D442G causes partial CETP deficiency $(54 \%$ reduction), resulting in a more moderate increase in HDL-C (mean of $96 \mathrm{mg} / \mathrm{dL}){ }^{12}$

Human CETP is a 476-residue, $74 \mathrm{kDa}$, hydrophobic glycoprotein primarily secreted by the liver and adipose tissue. ${ }^{13}$ CETP was first cloned in $1987 .{ }^{14}$ The structure of CETP allows formation of a tunnel with the opening on one end interacting with HDL and the other with a very low-density lipoprotein (VLDL), intermediate-density lipoprotein (IDL), or LDL particle. The hydrophobic central cavity of this tunnel is large enough to allow transfer of neutral lipids (eg, cholesteryl esters [CEs], triglycerides [TGs]) from donor to acceptor particles, but conformational changes may occur to accommodate larger lipoprotein particles. The concave surface of CETP matches the curvature of the HDL particles to which it is primarily bound in the bloodstream. ${ }^{15,16}$

The structure of CETP allows it to transfer CEs, TGs, and even phospholipids between apolipoprotein $\mathrm{B}$ (apoB) TG-rich particles (eg VLDL, chylomicrons), LDL and HDL based on their relative concentrations and composition; a process termed "heterotypic" transfer. When VLDL and chylomicrons are elevated, such as in diabetes, type IIB hyperlipidemia, or a postprandial state, these particles serve as the primary acceptor of $\mathrm{CE}$. In contrast during fasting, while levels of VLDL and chylomicrons are normal, or in patients with familial hypercholesterolemia, LDL becomes the primary acceptor of CE. The overall effect of CETP is a net transfer of $\mathrm{CE}$ from HDL to these apolipoprotein B (apoB)-containing particles and TG to HDL and LDL (Figure 1). An important driver of the transfer of $\mathrm{CE}$ from $\mathrm{HDL}$ to apoB-containing particles is the production of $\mathrm{CE}$ from free cholesterol within HDL by lecithin acetyltransferase (LCAT).${ }^{17}$ Additionally, CETP also serves to shuttle CE within HDL subclasses, termed "homotypic" transfer. Routine CETP activity assays typically only account for transfer of cholesteryl ester from donor particles (HDL3 or artificially engineered liposome) to apoB-containing 


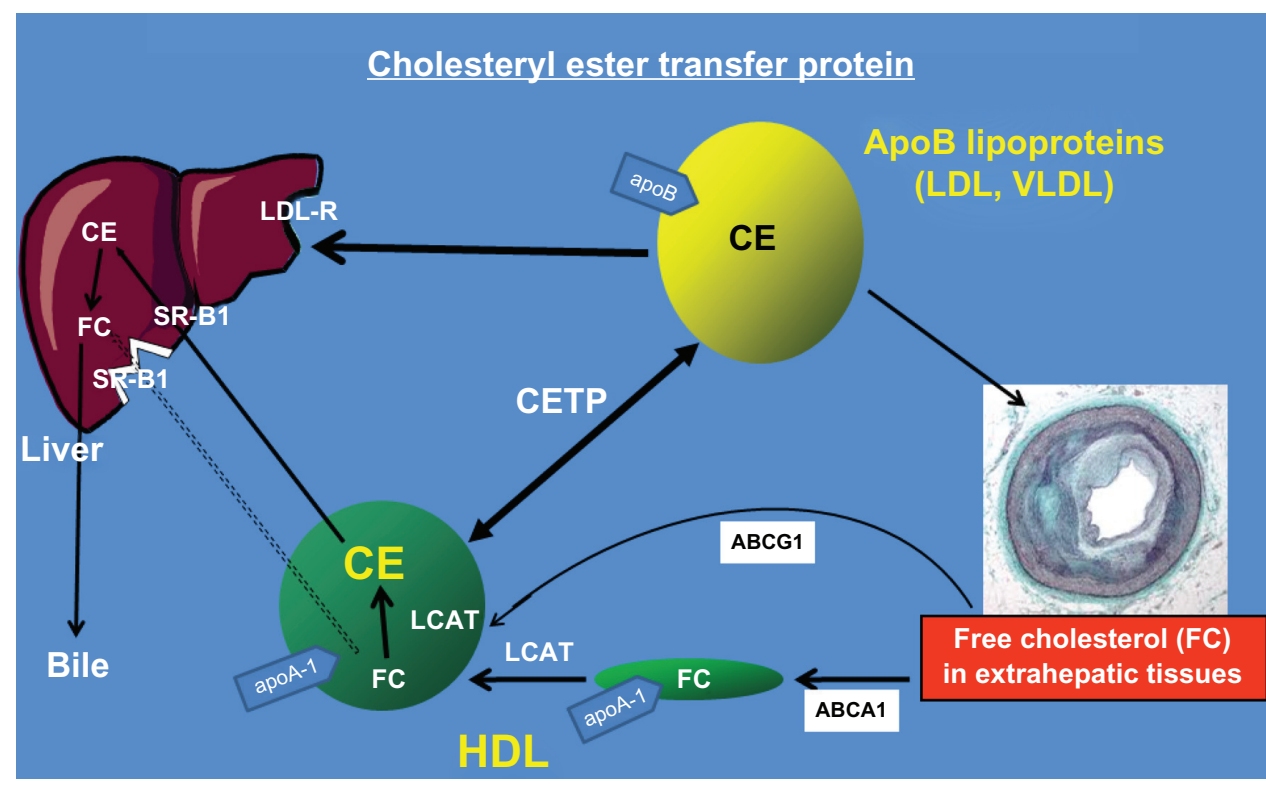

Figure I The role of CETP in reverse cholesterol transport.

Notes: Beginning in the peripheral tissues, free cholesterol is predominantly taken up by small "immature" HDL particles (eg, pre- $\beta$ - $H D L$ ) via the ABCAI transporter. Alternatively, it can be taken up by larger "mature" HDL particles (eg, HDL2) via the ABCGI transporter. LCAT converts free cholesterol into cholesteryl ester, which is then shuttled to apoB-lipoproteins (eg, LDL, VLDL) in exchange for triglycerides. Only a minority of cholesteryl ester is delivered directly to the liver by HDL via the SR-Bl; the majority is delivered indirectly to the liver by apoB-lipoproteins via the LDL recepter.

Abbreviations: CETP, cholesteryl ester transfer protein; HDL, high-density lipoprotein; ABCAI, ATP-binding cassette transporter AI; ABCGI, ATP-binding cassette transporter GI; LCAT, lecithin acetyltransferase; apoB, apolipoprotein B; LDL, low-density lipoprotein; VLDL, very low density lipoprotein; SR-BI, scavenger receptor-BI; FC, free cholesterol; CE, cholesteryl ester.

particles and do not take into account transfer from these donor particles to other HDL subclasses. ${ }^{18}$

In humans, homozygous CETP deficiency results in elevated levels of apoA-I due to markedly delayed apoA-I catabolism. There is an increased ratio of the large HDL2 particles to the smaller HDL particles (eg, pre $\beta$-HDL and HDL3c fractions) with a similar redistribution of apoAI. Complete CETP deficiency also results in extra-large apoE-rich HDL particles ( $>12 \mathrm{~nm})$, larger than in those with HALP or in normal subjects. ${ }^{19}$ This apoE enrichment most likely results as a consequence of the continued accumulation of CE in the core of these particles in CETP deficiency as well as an inability to transfer apoE to other larger particles. ${ }^{20}$

In homozygous CETP deficiency, apoB levels are decreased primarily through upregulation of LDL receptor activity. LDL particles are more heterogeneous in size with an increase in very small LDL particles, indicating that, as with HDL, CETP plays a role in LDL formation. Alteration of the normal LDL synthetic pathway together with the lack of a subsequent LDL-remodeling process may result in this pattern. ${ }^{21,22}$ Plasma LDL and total cholesterol levels tend to be lower in those with extra-large HDL particles. ${ }^{19}$

It is under debate whether the lipid modifications seen with CETP polymorphisms confer protection against atherosclerosis.
Interestingly, CETP activity differs across animal species; those species with no CETP activity seemingly have a reduced susceptibility to atherosclerosis. As in humans, animals that express CETP tend to have higher VLDL-C and LDL-C compared to HDL-C, whereas those with no CETP activity have a "reverse lipid profile" with higher HDL-C than LDL-C or VLDL-C. Mice, rats, and dogs have no significant CETP activity. Rabbits and monkeys have high CETP activity, while humans, hamsters, and chickens have intermediate CETP activity levels. This heterogeneity in the activity of CETP across species has complicated the interpretation of animal model findings and their implications to the biology of CETP and its inhibition in humans.

It is possible that CETP may be proatherogenic by decreasing HDL-C levels and increasing LDL-C levels. It is also possible that CETP may have an anti-atherogenic role by providing a mechanism to deliver CE from HDL to the liver, thereby increasing reverse cholesterol transport (a concept to describe cholesterol efflux from the entire periphery to the liver for fecal excretion). ${ }^{23}$ Data from transgenic mouse models expressing CETP have not been consistent. ${ }^{24-29}$ One theory serves to help explain the discrepant results of these mouse models. In normal mice, CEs are typically delivered to the liver by HDL via hepatic scavenger receptor-BI (SR-BI). However, in the setting of CETP expression, 
reverse cholesterol transport might actually increase despite a reduction in plasma HDL-C levels depending on the degree of CE uptake by the liver via the LDL receptor. In mice that have robust pathways for uptake of apoB-containing lipoproteins (eg, LDL), the expression of CETP, although it reduces HDL-C levels, might be expected to be anti-atherogenic. Conversely in mice that have markedly defective uptake of apoB-containing lipoproteins, expression of CETP might be expected to be proatherogenic. ${ }^{30}$

In contrast to mice, studies in humans have indicated that the majority of HDL CEs typically are returned to the liver by apoB-containing lipoproteins and not HDL. ${ }^{31}$ This observation suggested that in humans the hepatic SR-BI pathway may not be especially active and that CETP catalyzes the major pathway by which CEs are returned to the liver. Therefore, in humans, in the setting of impaired clearance of apoB-containing lipoproteins (eg, familial hypercholesterolemia, other genetic and environmental factors that reduce hepatic apoB-lipoprotein uptake), high CETP activity might be proatherogenic with deficiency (or inhibition) being relatively anti-atherogenic. On the other hand, in those with highly effective apoB-lipoprotein clearance (such as patients on high-dose statins), CETP might be relatively anti-atherogenic, with deficiency (or inhibition) being relatively proatherogenic. ${ }^{30} \mathrm{~A}$ recent analysis of the Pravastatin or Atorvastatin Evaluation and Infection Therapy - Thrombolysis in Myocardial Infarction 22 (PROVE IT - TIMI 22) trial evaluated the relationship between CETP mass (a surrogate for CETP activity) with the risk of recurrent coronary events and found that increased CETP mass levels were associated with decreased coronary events. Interestingly, this relationship was only present for those with LDL-C below the median (a marker of enhanced hepatic clearance). As introduced above, this finding might be explained by CETP actually increasing reverse cholesterol transport in those with enhanced CE uptake by LDL receptors. For those with normal or impaired CE uptake by LDL receptors, CETP might not improve reverse cholesterol transport. ${ }^{32}$ Because of the well-validated benefits of statins on CV events, outcomes trials will necessitate testing CETP inhibition on the background of statin therapy except in rare circumstances (eg, statin intolerance).

While the data from mouse models have been mixed, the results from hamster and rabbit models have consistently suggested CETP inhibition to be anti-atherogenic. In both normo- and hypercholesterolemic hamsters, use of a CETP-neutralizing antibody led to similar lipid modifications to those seen in homozygous CETP deficiency. ${ }^{33}$ In a rabbit model, immunization of cholesterol-fed rabbits with a CETP-based vaccine resulted in 24\% lower LDL-C and 42\% higher HDL-C, with a reduction in aortic atherosclerosis. ${ }^{34}$ In another rabbit study, reductions in atherosclerosis were also demonstrated with CETP inhibition using antisense oligonucleotides. ${ }^{35}$

One of the interesting questions in CETP deficiency is whether the HDL particles produced by potent CETP inhibition are functional. Regardless of whether reverse cholesterol transport is increased, the initial steps of cholesterol efflux from foam cells may be one of the key anti-atherogenic functions of HDL. ${ }^{5}$ Typically, the ATP-binding cassette (ABC) transporter ABCA1 promotes free cholesterol efflux from these cells to lipid-poor apoA-I and pre $\beta$-HDL. As mentioned earlier, in homozygous CETP deficiency these subclasses of HDL are diminished at the expense of larger HDL particles. ABCA1 is thought to poorly interact with these more "mature" HDL fractions. ABC transporter ABCG1, however, is activated by cholesterol accumulation within macrophages by the same transcription factors as ABCA1 and also plays a role in cholesterol efflux. In a study evaluating the preservation of this anti-atherogenic function of HDL in subjects with genetic CETP deficiency, it was found that macrophage cholesterol efflux is actually increased, primarily through ABCG1 (Figure 1). This increased efflux is related to the very high content of LCAT and apoE in these large HDL particles, presumably driving net cholesterol efflux by promoting cholesterol esterification. ${ }^{36}$ Additional studies have evaluated the effect of CETP deficiency on liver uptake of cholesteryl ester, an important downstream step in a reverse cholesterol transport. These studies suggest that there may be increased CE uptake via SR-BI as well as through a high affinity of large apoE-rich HDL for LDL receptors. ${ }^{20}$

Observational studies assessing the association of CETP deficiency with cardiovascular outcomes have been mixed. ${ }^{37-39}$ Recently, a meta-analysis established that three CETP genotypes were not only associated with decreased CETP activity and increased HDL but also with a lower risk of myocardial infarction (MI). For example, for each allele inherited, individuals with the TaqIB polymorphism had lower mean CETP activity (-8.6\%), higher mean HDL-C $(4.5 \%)$, higher mean apoA-I $(2.4 \%)$, and an odds ratio for coronary disease of 0.95 (95\% confidence intervals [CI], $0.92,0.99)$. Similar associations were found for the other two CETP genotypes. ${ }^{40}$ Subsequent studies have confirmed that genetic variants leading to reduced CETP activity and its corresponding anti-atherogenic lipid profile are associated with reduced atherosclerotic outcomes. ${ }^{41-43}$ 


\section{Lessons from torcetrapib}

Much of what is currently known about the impact CETP inhibitors have on atherosclerotic outcomes was derived from torcetrapib, the first CETP inhibitor to reach phase III trials. Torcetrapib is a 3,5-bis-trifluoromethyl-benzene derivative that forms stable, inactive complexes between CETP and lipoproteins (primarily HDL). Interestingly, while this decreases CETP activity, it results in an increase in CETP mass, presumably from accumulation of these complexes. ${ }^{44,45}$ It is unknown whether the accumulation of CETP has any biologic significance.

In Phase I-II studies, torcetrapib demonstrated dosedependent elevation of HDL-C (up to $\sim 60 \%$ ) and lowering of LDL-C ( $20 \%)$, both when administered as monotherapy or in combination with atorvastatin. ${ }^{46-48}$ Like other mechanisms of CETP inhibition, torcetrapib significantly reduced aortic atherosclerosis in a rabbit model ${ }^{49}$ Importantly, these early studies demonstrated an association of torcetrapib with increased blood pressure (BP) in both humans and animal models. The mechanism was not elucidated prior to launching the Phase III programs.

The Phase III studies included the outcomes study ILLUMINATE and three imaging trials, RADIANCE 1 and 2 and ILLUSTRATE. ILLUMINATE randomized 15,067 subjects with stable coronary heart disease (CHD) or CHD equivalent (eg, diabetes mellitus) 1:1 to atorvastatin or atorvastatin and torcetrapib $60 \mathrm{mg}$ daily. Despite torcetrapib-related LDL-C reductions of $24.9 \%$ and HDL-C elevations of $72.1 \%$, the trial was stopped early by the data monitoring committee due to a $25 \%$ increase (464 [6.2\%] vs $373[5.0 \%])$ in the primary endpoint (PEP) in the torcetrapib arm. Review of this composite endpoint demonstrated an increased risk for each of the components (ie, death from coronary heart disease, nonfatal MI [nonprocedure related], stroke, or hospitalization for unstable angina) as well as a $58 \%$ increase in death from any cause (93 vs 59 cases) $)^{50}$ (Figure 2). Although the deaths from cancer and infection were significantly elevated in the torcetrapib compared to the placebo group, overall reports of cancer and infection were not. The torcetrapib arm again demonstrated an increase in BP $(5.4 \mathrm{mmHg}$ increase in systolic BP), which post hoc exploratory analyses found to be associated with increased serum levels of aldosterone and serum electrolyte changes (reduced potassium, increased sodium and bicarbonate)..$^{50}$

The three imaging studies also randomized subjects to torcetrapib $60 \mathrm{mg}$ daily in addition to atorvastatin therapy. In RADIANCE 1, subjects with heterozygous familial hypercholesterolemia were evaluated for the primary endpoint of rate of change in maximum carotid intimal medial thickness. Despite similar lipid changes to ILLUMINATE, no significant difference was demonstrated in the primary endpoint at 2 years of follow-up. ${ }^{51}$ In RADIANCE 2 , subjects with mixed hyperlipidemia were evaluated for the same endpoint, but the trial was stopped early due to the results of ILLUMINATE; however, at almost 2 years of follow-up, no significant change was found between the arms. ${ }^{52}$ ILLUSTRATE used intravascular ultrasound to evaluate for a reduction in percent coronary atheroma volume; at 24 months the addition of torcetrapib to atorvastatin therapy failed to demonstrate a benefit compared to atorvastatin monotherapy. ${ }^{53}$ As a consequence of the findings of ILLUMINATE, Pfizer, the manufacturer of torcetrapib, closed down the torcetrapib development program.

The results of these trials raised the broad question of why the anticipated anti-atherogenic lipid modifications induced by CETP inhibition did not have a favorable effect on atherosclerotic plaque and if there were beneficial effects of torcetrapib, whether these might have been overcome by more deleterious ones. Scientific questions included: (1) Was the increase in BP enough to cause the increase in CV events? (2) Was CETP inhibition responsible for the elevation in BP or was this an "off-target" effect? (3) Were CETP-induced lipid changes truly anti-atherogenic? (4) What was the cause of the increase in mortality?

Beginning with the first two questions, it is unlikely that this modest BP elevation of itself resulted in a $25 \%$ increase in $\mathrm{CV}$ events without a significant increase in stroke (43 vs 40 in the torcetrapib and placebo arms, respectively, $P=0.74) .{ }^{50}$ Evaluation of the contribution of CETP inhibition to BP elevation included the following studies. Firstly, torcetrapib was shown to increase levels of cortisol and aldosterone by stimulating expression of the enzymes involved in the final step of their production in human adrenal cells. ${ }^{54}$ Secondly, torcetrapib treatment was found to increase circulating aldosterone and corticosterone in rats and BP elevation in rats, mice, and dogs, all species that lack CETP. Thirdly, CETP inhibitor compounds structurally different than torcetrapib were found not to result in these effects. ${ }^{55-57}$ Finally, an analysis of loss-of-function CETP single nucleotide polymorphisms, which reduce CETP activity and raise HDL-C, did not find an association with elevations in $\mathrm{BP}^{58}$ In total, these studies provided strong evidence that the aldosterone and BP elevations seen with torcetrapib were independent of its inhibition of CETP and were most likely due to off-target effects of the compound.

Further investigations have not yet clarified the mechanism of the torcetrapib-induced sustained blood effect. 


\section{Major CV events \\ (HR 1.25, $P=0.001$ )}

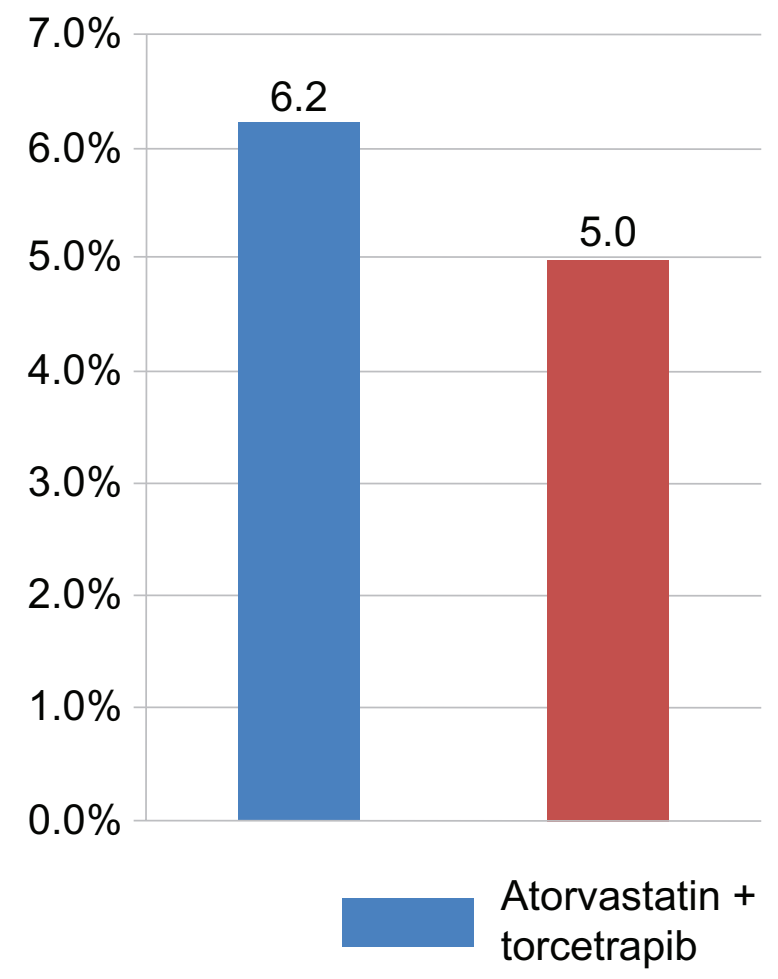

Death

(HR 1.58, $P=0.006$ )

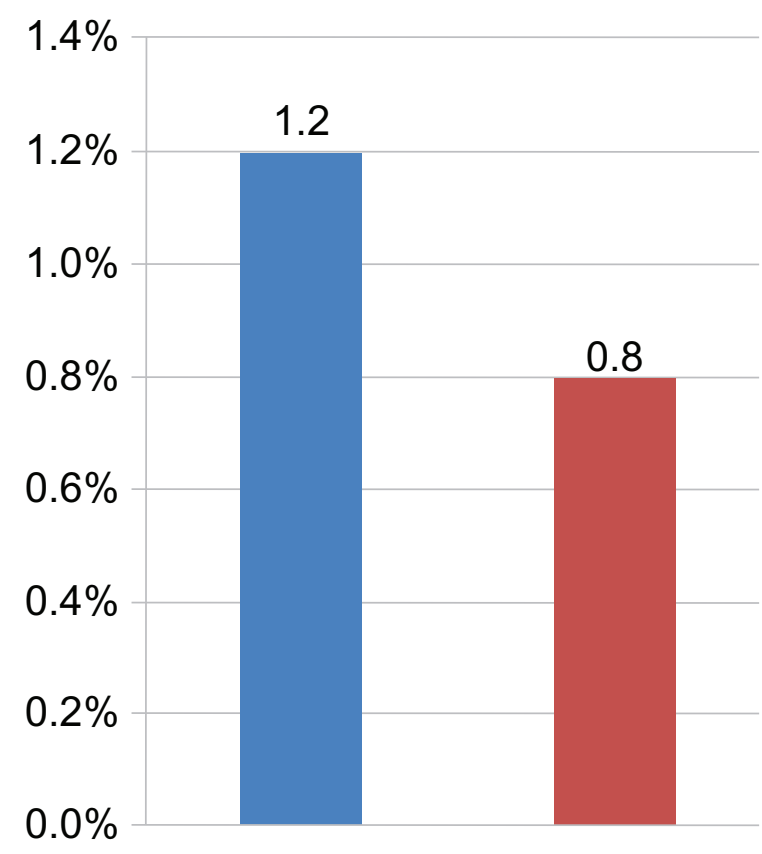

Atorvastatin monortheraphy

Figure 2 ILLUMINATE: risk of major outcomes with torcetrapib

Notes: ILLUMINATE was a randomized trial of torcetrapib, a cholesteryl ester transfer protein (CETP) inhibitor, in addition to atorvastatin ( $\mathrm{n}=7533)$ versus atorvastatin alone $(n=7534)$ in patients at high risk for CV events. (A) Comparison of patients who had the primary composite outcome: 373 patients in the atorvastatin-only group and 464 patients in the torcetrapib group. The primary outcome was the time to the first occurrence of a major cardiovascular (CV) event, a composite that included four components: death from coronary heart disease, nonfatal myocardial infarction (excluding procedure-related events), stroke, and hospitalization for unstable angina. (B) Comparison of patients who died from any cause during the study: 59 patients in the atorvastatin-only group and 93 patients in the torcetrapib group.

Experimental studies demonstrated that torcetrapib-induced aldosterone elevations are brief and unable to explain the sustained vasopressor response. Experiments with trilostane, an inhibitor of 3 $\beta$-hydroxysteroid dehydrogenase (enzyme needed for steroidogenesis), prevented the increase in aldosterone seen with torcetrapib but did not abolish the drug's vasopressor effect. ${ }^{59}$ Other experiments have demonstrated that the vasopressor effect was dependent upon the presence of intact adrenal glands, suggesting that other adrenal-derived mediator(s) may have been responsible. More recently, animal models have demonstrated that torcetrapib may also have had adverse effects on vascular endothelium; ${ }^{60-62}$ importantly, these effects appeared to be CETP independent as well.

In regards to the third question, the surprising failure of torcetrapib in the three imaging trials to stabilize or reduce atherosclerotic plaque despite a significant decrease in LDL-C and increase in HDL-C called into question whether torcetrapib-induced lipoprotein changes are anti-atherogenic. Reassurance came from studies that demonstrated reductions in postprandial formation and accumulation of atherogenic
TG-rich particles with torcetrapib therapy. ${ }^{63}$ In addition, torcetrapib-induced HDL demonstrated an increased ability to efflux cholesterol from macrophages; ${ }^{64}$ as in homozygous CETP deficiency, this correlated with the accumulation of apoE-rich HDL2 species and increased LCAT activity. ${ }^{66}$ In studies of reverse cholesterol transport in mouse and hamster models, torcetrapib demonstrated an increase in biliary and fecal sterol excretion. ${ }^{66,67}$

Further support for the functionality of torcetrapibinduced HDL came from post hoc analyses of the torcetrapib Phase III trials. In ILLUMINATE, those subjects with achieved HDL-C levels above the median had reduced CV events compared to those who did not. ${ }^{50}$ In ILLUSTRATE, an inverse association between HDL-C achieved and the primary endpoint of atheroma volume ( $\mathrm{r}=-0.17, P<0.001)$ was found. In addition, the highest quartile of HDL-C achieved (>86 mg/dL) demonstrated atheroma regression, suggesting that there may be a "threshold effect" to HDL-C elevation. ${ }^{68}$ To our knowledge, post hoc analyses of RADIANCE 1 and 2 have not demonstrated similar findings. 
A potential antidiabetic benefit of CETP inhibition was evaluated in a post hoc analysis of ILLUMINATE. In vitro data have suggested that HDL may have antidiabetic properties. ${ }^{69}$ In ILLUMINATE, those receiving torcetrapib had lower glucose levels, insulin levels, and measures of insulin resistance, although these results could not be definitely attributed to the torcetrapib-induced HDL increase. ${ }^{70}$

In regards to the last question, the increase in all-cause mortality seen in ILLUMINATE is still without a definitive explanation. Aside from CV death, cancer- and infectionrelated deaths were also increased. The explanation for this finding is uncertain, but it has been proposed that an excess in glucocorticoids may have decreased immunosurveillance, resulting in worse outcomes from infection and neoplasms. ${ }^{71}$ In summary, the ILLUMINATE failure, while raising appropriate concerns, did lead to encouragement for the potential of CETP inhibition if accomplished by "clean" CETP inhibitors (ie, without known off-target effects). These findings allowed the research community and manufacturers to move cautiously forward with other CETP inhibitors but only alongside a thorough re-evaluation of their safety, especially on BP, the vasculature, and the adrenal gland.

\section{Insights from other CETP inhibitors Dalcetrapib}

Unlike torcetrapib and anacetrapib, dalcetrapib is a benzenethiol derivative and binds at a different site than torcetrapib and anacetrapib. Dalcetrapib has a cysteine group, which allows formation of covalent bonds with various plasma proteins, including a disulfide bond with the cysteine 13 group of CETP. Dalcetrapib induces a time-dependent conformational change in CETP and similar to torcetrapib and anacetrapib, promotes the formation of a complex between CETP and HDL. ${ }^{18,45}$

The potency of dalcetrapib is significantly less than torcetrapib and anacetrapib. It reduces CETP activity by about $50 \%$ with a subsequent reduction in LDL-C of $<10 \%$ and an increase in HDL-C of 30\%-35\%. ${ }^{72}$ Like other methods of CETP inhibition, dalcetrapib was shown to reduce the development of atherosclerosis in cholesterol-fed rabbits. ${ }^{73}$ Unlike states of complete CETP deficiency or potent CETP inhibition, in vitro data suggested dalcetrapib had little effect on homotypic CE transfer (eg, not changing pre- $\beta$ HDL levels $)^{74}$ and did not result in the formation of large apoE-rich HDL2 particles, ${ }^{75}$ features that were proposed by some experts to perhaps be advantageous after the failure of ILLUMINATE.

Early Phase I and II studies of dalcetrapib demonstrated good tolerability without any effect on BP or the adrenal gland. ${ }^{76,77}$ In order to search for potential adverse vascular effects of the compound, a number of surrogate endpoint studies were initiated in parallel with a larger outcomes study. Safety and efficacy of dalcetrapib on atherosclerotic disease using novel non-invasive multimodality imaging (dal-PLAQUE) was an exploratory study that used position emission tomography and magnetic resonance imaging to evaluate vascular inflammation and remodeling. The results suggested that dalcetrapib did not increase carotid inflammation at 6 months or worsen atherosclerotic remodeling of the vasculature at 24 months. $^{78}$ Dal-VESSEL evaluated the BP and vascular effects of dalcetrapib using 24-hour ambulatory BP monitoring and brachial flow-mediated dilatation. The results confirmed dalcetrapib did not increase BP or reduce nitric oxide (NO)-dependent endothelial function. ${ }^{79}$

The Phase III dal-OUTCOMES trial randomized 15,871 patients with recent acute coronary syndrome (ACS) to dalcetrapib $600 \mathrm{mg}$ daily versus placebo. The primary efficacy measure was time-to-first occurrence of CHD death, nonfatal acute MI, and unstable angina requiring hospital admission, resuscitated cardiac arrest, or atherothrombotic stroke. ${ }^{80}$ In May 2012, at a prespecified interim analysis of $71 \%$ of the projected target endpoints, the data monitoring committee recommended stopping the trial for futility. Based on this recommendation, this trial along with the remaining dalcetrapib trials was stopped by Roche, the drug manufacturer and trial sponsor. ${ }^{81}$

The stopping of dal-OUTCOMES was disappointing to proponents of the beneficial effects of CETP inhibition and pharmacologic HDL-C raising. Since dalcetrapib only significantly modifies HDL-C, the trial was powered purely based on the anticipated benefits of HDL-C augmentation on lowering CV risk. Surprisingly, dal-OUTCOMES demonstrated no relationship between baseline HDL-C or increases in HDL-C and the risk of CV events. In regards to the population, the trial cohort was enrolled after recent ACS (median 61 days from index event) and followed for up to 3 years. Some have suggested that the benefits of HDL augmentation may have been difficult to detect in the setting of a recent ACS. If the timing of enrollment was the culprit, one might expect a signal for benefit to develop as the cohort stabilized; however, no signal for incremental benefit was present, even for those subjects with the longest follow-up. In retrospect, dal-OUTCOMES may indicate that either (or both) dramatic HDL-C elevations or significant reductions in atherogenic particles, including LDL-C, apoB, non-HDL-C, and $\mathrm{Lp}(\mathrm{a})$, are needed to demonstrate clinical benefit from CETP inhibition. 
Reassuringly, the safety profile of dalcetrapib was acceptable, suggesting that at least moderate CETP inhibition is not associated with any clinically obvious concerns. Importantly, a minor elevation of systolic $\mathrm{BP}(0.6 \mathrm{mmHg})$ was found in the dalcetrapib arm. ${ }^{82}$ Since dalcetrapib is structurally different than torcetrapib, these findings revive the discussion of whether there are unknown biologic consequence(s) of CETP inhibition. Although not associated with any clinical safety concerns, mechanistic investigations for a connection to CETP inhibition are warranted. In light of the torcetrapib and dalcetrapib experiences, it is still unknown whether near-complete inhibition with a "clean" CETP inhibitor might be beneficial.

\section{Evacetrapib}

The latest CETP inhibitor evacetrapib is a novel benzazepinebased compound. The Phase II results demonstrated potent dose-dependent lipid modification; the effect on Lp(a) was not reported (Table 1) ${ }^{83,96}$ In addition, the compound demonstrated good tolerance without a significant increase in adverse events, including aldosterone, cortisol, or BP elevation. The Phase III outcomes study ACCELERATE (NCT01687998) has recently begun an anticipated enrollment of 11,000 subjects for an average follow-up of approximately 2 years. ACCELERATE will enroll a higher risk coronary artery disease (CAD) cohort (within 1 year of index ACS) with a broader primary composite endpoint, including CV death, MI, stroke, coronary revascularization, or hospitalization for unstable angina. ACCELERATE will test a new formulation of evacetrapib that is anticipated to have similar lipid-modifying effects as the $500 \mathrm{mg}$ dose tested in the Phase II study (Table 2). ${ }^{84}$

\section{Anacetrapib}

Anacetrapib is a 3,5-bis-trifluoromethyl-benzene derivative with similar binding properties to CETP as torcetrapib. The compound was developed when it was found that a substitution modification of the oxazolidinone ring increased its potency for CETP inhibition in a transgenic mouse model. ${ }^{85}$ In terms of its pharmacokinetics and pharmacodynamics, anacetrapib is rapidly absorbed with a time-to-peak plasma concentration of about 4 hours. The oral bioavailability of anacetrapib is poor, with only about $20 \%$ being absorbed; however at this exposure, LDL-C is reduced up to $40 \%$ and HDL-C increased up to $140 \%$. It is recommended that anacetrapib be taken with food (ie, low-fat diet) to increase drug exposure (and efficacy) as well as compliance. ${ }^{86}$

Anacetrapib is highly protein bound (eg, CETP) in the plasma $(>99.5 \%)$. It is cleared by oxidative metabolism via
Cytochrome P450 3A4 (CYP3A4) with excretion of the metabolites via the biliary/fecal route. Only a trace amount is eliminated by urinary excretion. ${ }^{87}$ Importantly, while anacetrapib is a sensitive CYP3A4 substrate, anacetrapib neither inhibits nor induces CYP3A4 activity. No meaningful interactions have been found between anacetrapib and simvastatin, digoxin, or warfarin. ${ }^{86}$ Anacetrapib in part to its redistribution to adipose tissue has a long terminal half-life. ${ }^{88}$

Phase I-II studies of anacetrapib demonstrated that higher doses result in near-complete inhibition of CETP activity with resultant LDL-C and HDL-C levels similar to those seen in homozygous CETP deficiency (Table 1)..$^{89,90}$ Lipid changes include: (1) decreased VLDL-C, IDL-C, and LDL-C; (2) TG enrichment and CE depletion within apoBcontaining particles; (3) decreased medium and smaller LDL particles with an increase in very small LDL particles; and (4) increased HDL2 and total HDL cholesterol and particle concentrations with absolute increases in apoA-I, apoA-II, apoE, and apoC-III (only apoC-III, however, demonstrates a percentage mass/particle increase). Additionally, decreases in $\mathrm{Lp}$ (a) up to $43 \%$ were found..$^{91}$ Using the results of these studies, a model-based strategy was employed to decide which dose would be used as the program advanced into larger clinical studies. The $100 \mathrm{mg}$ daily dose was selected as it is the minimum dose necessary to achieve the near maximal effects on HDL-C and LDL-C (ie, the flat part of the dose-response curve). ${ }^{92}$

In light of the torcetrapib experience, anacetrapib was tested and demonstrated no effect on adrenal steroid levels in vitro or in animal models. ${ }^{55}$ Functional assessments of anacetrapib-induced HDL demonstrated: (1) enhanced cholesterol efflux properties from macrophages possibly driven by the increased apoE and LCAT concentration; and (2) ability to suppress macrophage toll-like receptor 4-mediated inflammatory responses in the same concentration-dependent manner as HDL particles from untreated subjects. ${ }^{93}$ More recently, a hamster model of reverse cholesterol transport demonstrated the ability of anacetrapib to deliver CE to the liver for biliary-to-feces excretion. ${ }^{94}$

The DEFINE trial was a randomized, double-blind, placebo-controlled study to further assess the safety of anacetrapib in patients with $\mathrm{CHD}$ or CHD equivalents. A Bayesian approach was applied to determine the predictive probability (confidence) to dismiss a torcetrapib-like $25 \%$ increase in CV events as observed in ILLUMINATE, in addition to a long list of prespecified safety parameters. Lipid entry criteria were LDL-C $50-100 \mathrm{mg} / \mathrm{dL}$ while on statin therapy with or 
Table I Lipid-modifying efficacy of anacetrapib and evacetrapib

\begin{tabular}{|c|c|c|c|}
\hline & Anacetrapib $100 \mathrm{mg}$ & Evacetrapib $100 \mathrm{mg}$ & Evacetrapib $500 \mathrm{mg}^{\mathrm{a}}$ \\
\hline \multicolumn{4}{|l|}{ TC } \\
\hline Relative (monotherapy) \% & --- & $10(6$ to 13$)$ & II (5 to I6) \\
\hline Relative (on stain) \% & 14 & 12 to $16^{b}$ & Not evaluated \\
\hline \multicolumn{4}{|l|}{ LDC-Cc } \\
\hline Relative (monotherapy) \% & --- & $-26(-33$ to -19$)$ & $-40(-47$ to -33$)$ \\
\hline Relative (on stain) \% & -40 & -14 to $-11^{b}$ & Not evaluated \\
\hline \multicolumn{4}{|l|}{ HDL-C } \\
\hline Relative (monotherapy) \% & --- & $98(85$ to III) & 132 (| | 9 to | 45$)$ \\
\hline Relative (on stain) \% & 138 & 79 to $89^{b}$ & Not evaluated \\
\hline \multicolumn{4}{|l|}{ TG } \\
\hline Relative (monotherapy) \% & --- & $-12(-24$ to -1$)$ & $-20(-32$ to -8$)$ \\
\hline Relative (on stain) \% & -7 & -8 to $I^{b}$ & Not evaluated \\
\hline \multicolumn{4}{|l|}{ Аров } \\
\hline Relative (monotherapy) \% & --- & $-16(-22$ to -11$)$ & $-26(-3 \mid$ to -20$)$ \\
\hline Relative (on stain) \% & -21 & -7 to $-5^{b}$ & Not evaluated \\
\hline \multicolumn{4}{|l|}{ ApoAl } \\
\hline Relative (monotherapy) \% & --- & 36 (30 to 42$)$ & 50 (44 to 57$)$ \\
\hline Relative (on stain) \% & 45 & 29 to $34^{b}$ & Not evaluated \\
\hline \multicolumn{4}{|l|}{ Lp(a) (nmol/L) } \\
\hline Relative (on stain) \% & -36 & Not published & Not published \\
\hline
\end{tabular}

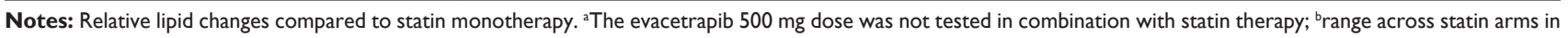
Phase II study; 'these LDL-C measurements were performed using the Freidewald equation in DEFINE versus an enzymatic method in the Phase II evacetrapib study. Abbreviations: LDL-C, low-density lipoprotein cholesterol; DEFINE, Determining the EFficacy and tolerability of CETP INhibition with AnacEtrapib; TC, total cholesterol; HDL-C, high-density lipoprotein cholesterol; TG, triglyceride; Apo B, apolipoprotein B; Apo Al, apolipoprotein Al; Lp(a), lipoprotein(a).

without other lipid-lowering therapy, HDL-C $<60 \mathrm{mg} / \mathrm{dL}$, and TG $<400 \mathrm{mg} / \mathrm{dL}$. Subjects were assigned to either anacetrapib $100 \mathrm{mg}$ daily or placebo for a 76-week treatment period followed by a 12-week, off study, drug "reversal" period. The study drug was discontinued for those patients achieving an LDL-C $<25 \mathrm{mg} / \mathrm{dL}$. Investigators were prompted to increase the lipid-lowering therapy for those with an LDL-C >115 mg/dL. Secondary to anacetrapib's robust lowering of atherogenic apoB-containing lipoproteins, the primary endpoint was LDL-C at 24 weeks. ${ }^{95}$

In DEFINE, 1623 subjects were randomized to either anacetrapib or placebo. For 142 patients in the anacetrapib arm and one patient in the placebo arm, treatment was discontinued due to LDL-C $<25 \mathrm{mg} / \mathrm{dL}$. Follow-up and safety assessments were achieved in $>99 \%$ of subjects in both arms. The primary endpoint, LDL-C decreased from 81 to $45 \mathrm{mg} / \mathrm{dL}$ in the anacetrapib arm as compared to 82 to $77 \mathrm{mg} / \mathrm{dL}$ in the placebo $\operatorname{arm}(39.8 \%$ reduction with anacetrapib compared to placebo). HDL-C increased from 41 to $101 \mathrm{mg} / \mathrm{dL}$ in the anacetrapib arm as compared to 40 to $46 \mathrm{mg} / \mathrm{dL}$ in the placebo arm (138.1\% increase with anacetrapib compared to placebo) (Figure 3). ${ }^{96}$ Table 1 provides a full listing of the lipid effects in DEFINE.

Importantly, CETP inhibitor studies to date have largely reported calculated LDL-C using the Freidewald (FR) equation. A recent study demonstrated that in anacetrapibtreated patients, the FR equation overestimates LDL-C reduction by $12.2 \mathrm{mg} / \mathrm{dL}$ compared to the $\beta$-quantification reference method. It is believed that CETP inhibitor-induced alterations in the composition of lipoproteins, renders the division of TG by 5 in the FR equation no longer valid. The LDL-C measurements reported in DEFINE were calculated by the FR equation, and thus anacetrapib-induced LDL-C reductions are likely to be somewhat less than $40 \%$. The LDL-C measurements reported in DEFINE were calculated by the FR equation, and thus the true LDL-C reductions are somewhat less. The Phase II study of evacetrapib measured LDL-C by using an enzymatic method; enzymatic assays are expected to produce LDL-C values closer to those of the $\beta$-quantification method. ${ }^{97}$

In terms of safety endpoints, anacetrapib demonstrated no increase in side effects (including myalgia), drug-related adverse effects, adverse events leading to drug discontinuation, or other important safety endpoints, such as BP, electrolyte, aldosterone, creatinine kinase, or transaminase levels. A very small increase in C-reactive protein of undetermined significance was seen with anacetrapib, which notably was also reported with torcetrapib and dalcetrapib in their Phase III studies. It is unknown whether this is a class effect as the small sample size in the evacetrapib Phase II study limits evaluation of small C-reactive protein changes. Most importantly, the prespecified adjudicated $\mathrm{CV}$ events occurred in 16 patients treated with anacetrapib $(2.0 \%)$ and 21 patients $(2.6 \%)$ treated 
Table 2 Phase III outcomes study comparisons.

\begin{tabular}{|c|c|c|}
\hline & Anacetrapib & Evacetrapib \\
\hline Dose & 100 mg daily & 130 mg dailya \\
\hline Sample size & 30,000 & 11,000 \\
\hline Run-in-period & Yes $(8-12$ weeks $)$ & No formal run-in period \\
\hline Background statin & Atorvastatin 20 to $80 \mathrm{mg}$ (except China: $10 \mathrm{mg}$ or $20 \mathrm{mg}$ ) & Physician/investigator discretion \\
\hline $\begin{array}{l}\text { Lipid treatment/levels } \\
\text { (protocol specified) }\end{array}$ & $\begin{array}{l}\text { I. Screening cutoff: } \mathrm{TC}<135 \mathrm{mg} / \mathrm{dL} \\
\text { 2. Randomization cutoff: } \mathrm{TC} \leq 155 \mathrm{mg} / \mathrm{dL} \\
\text { 3. Tolerate study atorvastatin during run-in period (LDL-C } \\
\text { lowering efficacy of dose assigned at screening at least as } \\
\text { effective as prior therapy) }\end{array}$ & $\begin{array}{l}\text { I. Tolerate statin therapy for } \geq 30 \text { days prior to } \\
\text { screening (unless documented stain statin intolerance/ } \\
\text { contraindication) } \\
\text { 2. Screening cutoff LDL-C no more than } 10 \mathrm{mg} / \mathrm{dL} \text { above the } \\
\text { investigator-chosen target (either } 100 \mathrm{mg} / \mathrm{dL} \text { or } 70 \mathrm{mg} / \mathrm{dL} \text { ) } \\
\text { or } \\
\text { Screening LDL-C above target but subject on maximally } \\
\text { tolerated statin or with documented statin intolerance/ } \\
\text { contraindication }\end{array}$ \\
\hline \multirow[t]{5}{*}{ Med $\mathrm{Hx}$ inclusions } & I. Age $\geq 50 \mathrm{yrs}$ & I. Age $\geq 18$ yrs \\
\hline & 2. Hx of $\mathrm{Ml}$ ( $>4$ wks prior to screening) & 2. Hx of ACS ( 30 to 365 days) \\
\hline & 3. Stroke or carotid/vertebral revascularization & 3. Cerebrovascular atherosclerotic disease \\
\hline & 4. PAD repair/revascularization & 4. $P A D$ \\
\hline & 5. DM with symptomatic CAD & 5. DM with documented $C A D$ \\
\hline Med Hx exclusions & Similar & Similar \\
\hline PEP & Coronary death, $\mathrm{Ml}$, or coronary revascularization & $\begin{array}{l}\text { CV death, MI, stroke, coronary revascularization, or } \\
\text { hospitalization for UA }\end{array}$ \\
\hline Study duration & $\begin{array}{l}\text { I. June } 20 \mathrm{II} \text {-Jan } 20 \mathrm{I} 7 \text { (median } \geq 4 \mathrm{yrs} \text { ) } \\
\text { 2. } \geq 1900 \text { primary endpoint events } \\
\text { 3. } \geq 950 \text { coronary death or } \mathrm{Ml} \text { events }\end{array}$ & I. Oct 2012 -Sept 2015 ( $\leq 4$ yrs maximum follow-up) \\
\hline
\end{tabular}

Notes: ${ }^{a}$ The formulation of the evacetrapib tablet tested in the Phase III program has been modified from that used in the Phase II program; the lipid-modifying efficacy of the dose/formulation is likely similar to that of the prior formulation at the $500 \mathrm{mg}$ dose. Comparison of REVEAL ${ }^{100}$ and ACCELERATE. ${ }^{84}$

Abbreviations: REVEAL, Randomized EValuation of the effects of anacetrapib through lipid-modification; ACCELERATE, a study of evacetrapib in high-risk vascular disease; TC, total cholesterol; MI, myocardial infarction; PAD, peripheral arterial disease; DM, diabetes mellitus; CAD, coronary artery disease; LDL-C, low-density lipoprotein cholesterol; ACS, acute coronary syndrome; CV, cardiovascular; UA, unstable angina.

with placebo. The Bayesian analysis provided a $94 \%$ predictive probability that anacetrapib is not associated with the rate of adverse $\mathrm{CV}$ events reported with torcetrapib. ${ }^{96}$

Prespecified subgroup analyses demonstrated a fairly uniform response to anacetrapib except in black subjects in whom drug levels were lower with a diminished LDL-C ( $25 \%$ reduction) and HDL-C response ( $80 \%$ increase). This finding will require confirmation due to the small number of black subjects $(n=34)$ enrolled in DEFINE. ${ }^{98}$

In those allocated to anacetrapib, persistent plasma levels were found 12 weeks after drug cessation with attenuated but persistent lipid effects; LDL-C (-19\%), non-HDL-C $(-18 \%)$, Apo B $(-10 \%)$, HDL-C $(+73 \%)$ and ApoA-I $(+25 \%) .{ }^{99}$ Follow-up studies are in progress to determine the pharmacokinetic and pharmacodynamic features of longterm anacetrapib administration.

Based off the encouraging efficacy and safety results of DEFINE, the Phase III outcomes study REVEAL (NCT01252953) is currently underway (Figure 4 and Table 2). REVEAL is expected to randomize 30,000 stable patients with prior MI, diabetes mellitus with coronary artery disease, peripheral arterial revascularization, and/or stroke to either placebo or anacetrapib in addition to atorvastatin therapy. The primary endpoint is a composite of coronary death, MI, or coronary revascularization. The trial is anticipated to finish in 2017 with an anticipated median follow-up duration of 4 years (Table 2). ${ }^{100}$ There are important differences between the study designs and patient populations in REVEAL and ACCELERATE. Both trials are expected to enroll patients predominantly under the inclusion criterion of a prior coronary event. REVEAL will enroll a slightly lower risk population since patients greater than 1 year from their last MI are eligible; whereas in ACCELERATE these patients must be enrolled within 1 year of their last ACS. It is expected that the REVEAL population will also have lower starting LDL-C levels, both because statin-intolerant subjects will not be enrolled and because of more stringent lipid entry criteria. The final major difference is that the primary endpoint in REVEAL is focused on coronary events, while ACCELERATE has a broader primary endpoint. A broader primary endpoint along with a slightly higher risk population will allow for a shorter follow-up duration and much smaller sample size in ACCELERATE.

Together REVEAL and ACCELERATE will provide more definitive answers on potent CETP inhibition in reduc- 

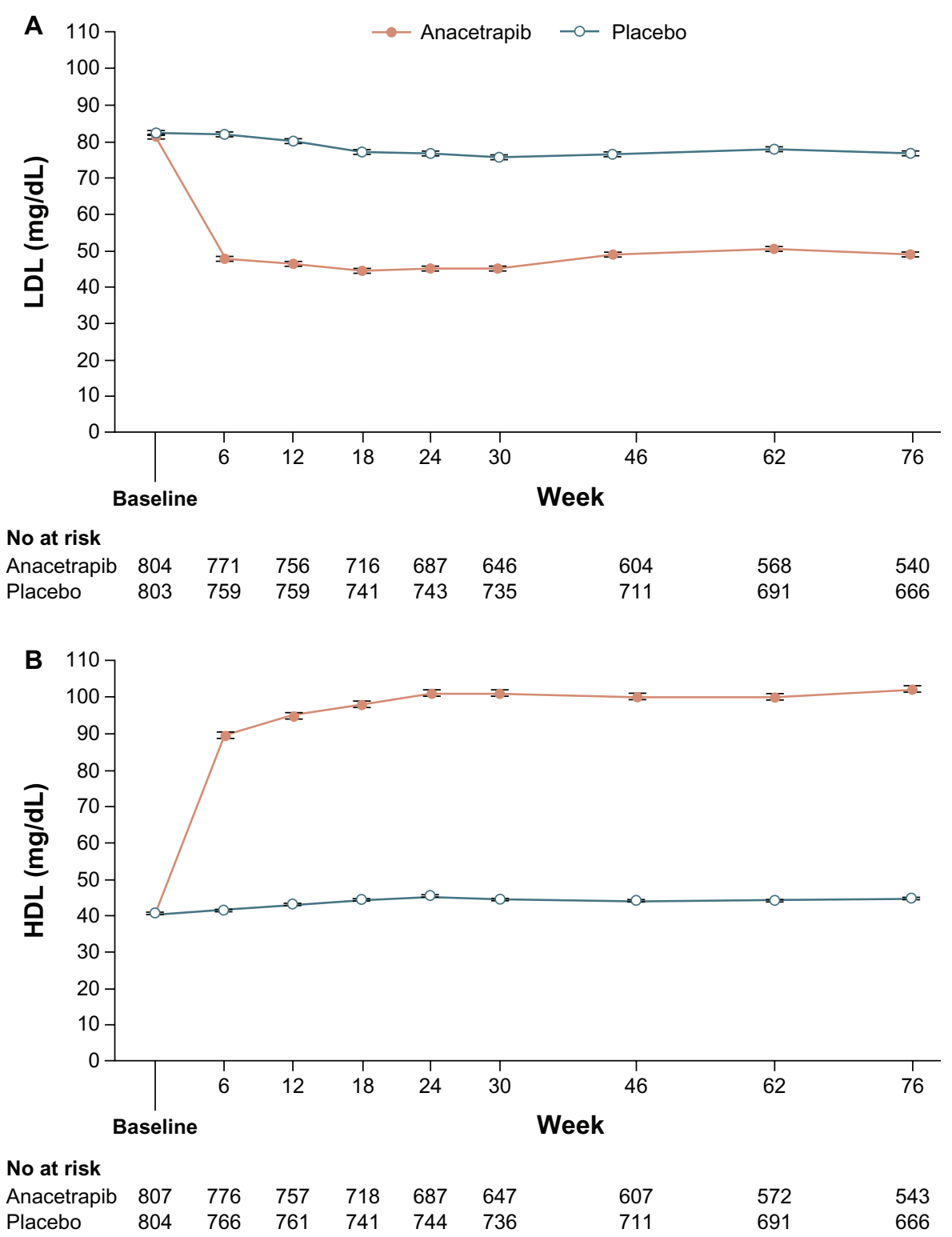

Figure 3 Change in cholesterol levels in DEFINE.

Notes: LDL and HDL cholesterol levels in the anacetrapib and placebo groups from baseline to week 76 . Horizontal bars indicate standard errors. To convert the values for LDL and HDL cholesterol to millimoles per liter, multiply by 0.02586 .

From Cannon CP, Dansky HM, Davidson M, et al. Design of the DEFINE trial: Determining the EFficacy and tolerability of CETP INhibition with AnacEtrapib. Am Heart J. 158(4):513-519. e3. ${ }^{95} \odot 2009$ Massachusetts Medical Society. Adapted with permission from Massachusetts Medical Society.

Abbreviations: DEFINE, Determining the EFficacy and tolerability of CETP INhibtion with AnacEtrapib; LDL, low-density lipoprotein; HDL, high-density lipoprotein.

ing CV outcomes and its long-term safety. Unlike DEFINE, REVEAL will not mandate discontinuation of anacetrapib for LDL-C $<25 \mathrm{mg} / \mathrm{dL}$ and thus will provide further information on treatment to very low LDL-C levels. It is unknown to the authors whether ACCELERATE will discontinue evacetrapib at a minimum threshold.

\section{Conclusion}

The history of CETP inhibition has had a turbulent beginning with the failure of its first two compounds in large outcomes trials. Despite this, observational and experimental evidence indicate that CETP remains a valid target and that the lipid changes resulting from its inhibition may be protective. The biology of CETP inhibition is complex, and questions remain regarding which lipid changes (eg, reductions in LDL and Lp(a), increases in HDL) are most likely to be important and whether there are still unknown effects that may negate any overall clinical benefit. In addition, if potent CETP inhibition is found to be beneficial, it is still unclear whether this effect will be homogeneous or vary based on individual metabolism.

Anacetrapib and evacetrapib are now poised to test the hypothesis that CETP inhibition may reduce atheroscle- 


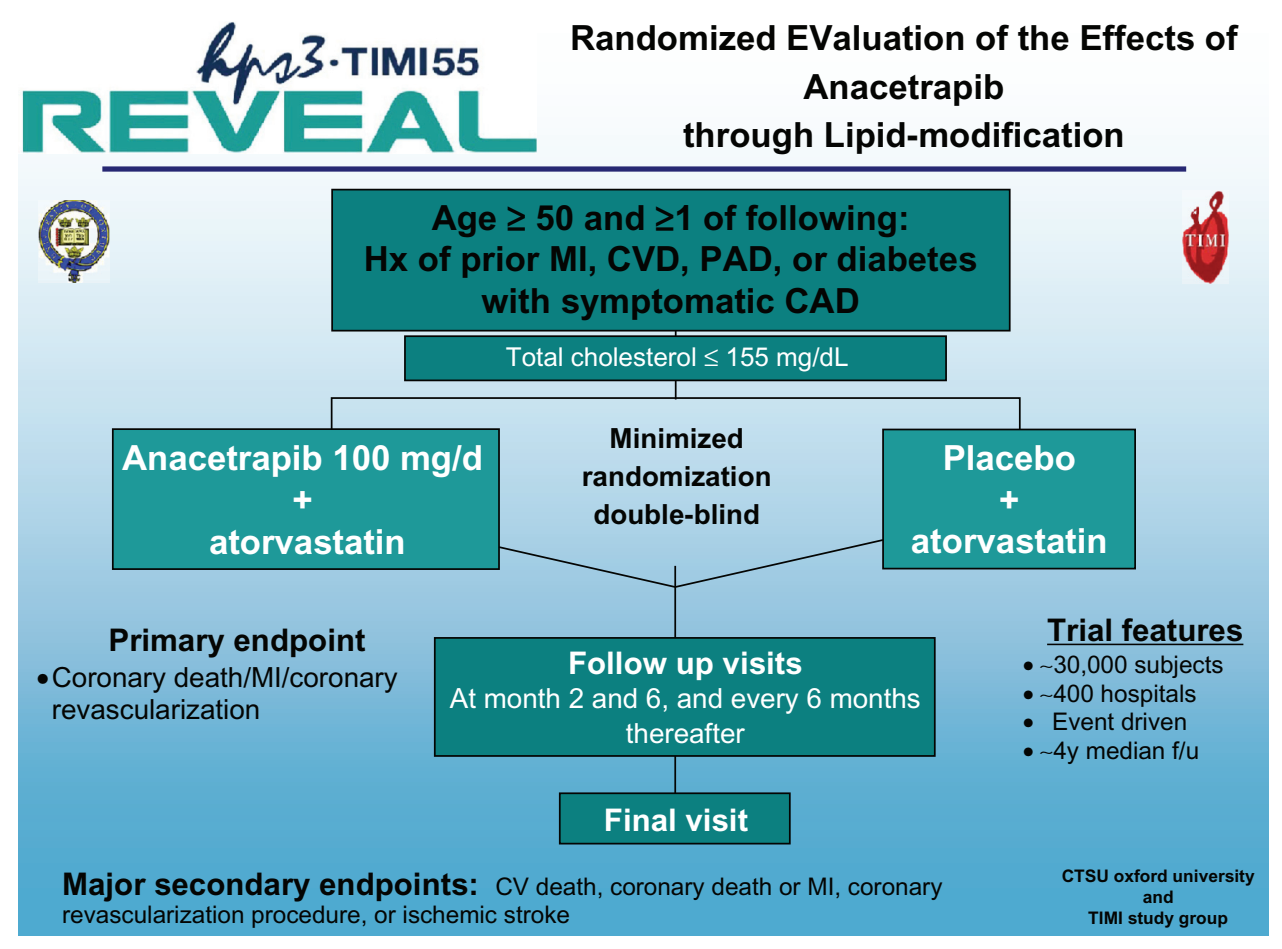

Figure 4 REVEAL trial design

Abbreviation: MI, myocardial infarction; CVD, cerebrovascular disease; PAD, peripheral arterial disease; CAD, coronary artery disease; ACS, acute coronary syndrome; CV, cardiovascular; REVEAL, Randomized Evaluation of the Effects of Anacetrapib through Lipid-modification.

rotic outcomes. In contrast to torcetrapib and dalcetrapib, these compounds may have advantageous features that result in clinical efficacy with an acceptable safety profile. Compared to torcetrapib, anacetrapib has consistently demonstrated no off-target effects. In addition, the results of DEFINE suggested a reduction in coronary revascularization with anacetrapib, potentially indicating a benefit for coronary atherosclerosis. Compared to dalcetrapib and torcetrapib, anacetrapib produces greater reductions in LDL-C, non-HDL-C, and Lp(a), which may result in a significant clinical effect, regardless of the benefit of HDL-C elevation. Experimental data do, however, support that anacetrapib-induced HDL (especially the apoE-rich HDL2 particles) may have an enhanced ability for reverse cholesterol transport without any known adverse effects. Importantly, if a threshold effect for HDL-C augmentation exists, the vast majority of patients taking anacetrapib would be expected to cross it.

The risk of a recurrent $\mathrm{CV}$ event in patients with a prior atherosclerotic remains unacceptably high. Despite a difficult beginning for the class of CETP inhibitors, anacetrapib and evacetrapib hold promise as future therapies for patients with atherosclerosis as well as to answer important questions regarding the role of CETP in the biology of lipid metabolism and atherosclerosis.

\section{Disclosure}

Dr Cannon is a member of the advisory boards of and has received grant support from Alnylam, Bristol-Myers Squibb, Pfizer, and CSL Behring; has received grant support from Accumetrics, AstraZeneca, Essentialis, GlaxoSmithKline, Merck, Regeneron, Sanofi, and Takeda; and is a clinical advisor to Automated Medical Systems. All other authors have reported that they have no relationships relevant to the contents of this paper.

\section{References}

1. Cholesterol Treatment Trialists' (CTT) Collaboration, Baigent C, Blackwell L, et al. Efficacy and safety of more intensive lowering of LDL cholesterol: A meta-analysis of data from 170,000 participants in 26 randomised trials. Lancet. 2010;376(9753): $1670-1681$.

2. Cannon $\mathrm{CP}$, Braunwald $\mathrm{E}, \mathrm{McCabe} \mathrm{CH}$, et al. Intensive versus moderate lipid lowering with statins after acute coronary syndromes. $N$ Engl J Med. 2004;350(15):1495-1504.

3. Steinberg D. The rationale for initiating treatment of hypercholesterolemia in young adulthood. Curr Atheroscler Rep. 2013;15(1):296.

4. Gordon T, Castelli WP, Hjortland MC, Kannel WB, Dawber TR. High density lipoprotein as a protective factor against coronary heart disease. the framingham study. Am J Med. 1977;62(5):707-714.

5. Vergeer M, Holleboom AG, Kastelein JJ, Kuivenhoven JA. The HDL hypothesis: Does high-density lipoprotein protect from atherosclerosis? J Lipid Res. 2010;51(8):2058-2073.

6. AIM-HIGH Investigators, Boden WE, Probstfield JL, et al. Niacin in patients with low HDL cholesterol levels receiving intensive statin therapy. N Engl J Med. 2011;365(24):2255-2267. 
7. Armitage J, Baigent C, Chen Z, Landray M. Treatment of HDL to reduce the incidence of vascular events HPS2-THRIVE. Available from: http:// clinicaltrials.gov/ct2/show/NCT00461630. Updated 2010. Accessed February 1, 2012.

8. Merck announces HPS2-THRIVE study of TREDAPTIVE (extendedrelease Niacin/Laropriprant) did not achieve primary endpoint. Available from: http://www.mercknewsroom.com/press-release/ prescription-medicine-news/merck-announces-hps2-thrive-studytredaptive-extended-relea. Updated 2012. Accessed December 31, 2012.

9. Tsimikas S, Hall JL. Lipoprotein(a) as a potential causal genetic risk factor of cardiovascular disease: A rationale for increased efforts to understand its pathophysiology and develop targeted therapies. $J \mathrm{Am}$ Coll Cardiol. 2012;60(8):716-721.

10. Brown ML, Inazu A, Hesler CB, et al. Molecular basis of lipid transfer protein deficiency in a family with increased high-density lipoproteins. Nature. 1989;342(6248):448-451.

11. Inazu A, Brown ML, Hesler CB, et al. Increased high-density lipoprotein levels caused by a common cholesteryl-ester transfer protein gene mutation. N Engl J Med. 1990;323(18):1234-1238.

12. Ohtani R, Inazu A, Noji Y, et al. Novel mutations of cholesteryl ester transfer protein (CETP) gene in Japanese hyperalphalipoproteinemic subjects. Clin Chim Acta. 2012;413(5-6):537-543.

13. Chapman MJ, Le Goff W, Guerin M, Kontush A. Cholesteryl ester transfer protein: At the heart of the action of lipid-modulating therapy with statins, fibrates, niacin, and cholesteryl ester transfer protein inhibitors. Eur Heart J. 2010;31(2):149-164.

14. Drayna D, Jarnagin AS, McLean J, et al. Cloning and sequencing of human cholesteryl ester transfer protein cDNA. Nature. 1987;327(6123): 632-634.

15. Qiu X, Mistry A, Ammirati MJ, et al. Crystal structure of cholesteryl ester transfer protein reveals a long tunnel and four bound lipid molecules. Nat Struct Mol Biol. 2007;14(2):106-113.

16. Zhang L, Yan F, Zhang S, et al. Structural basis of transfer between lipoproteins by cholesteryl ester transfer protein. Nat Chem Biol. 2012;8(4):342-349.

17. Barter PJ, Brewer HB Jr, Chapman MJ, Hennekens CH, Rader DJ, Tall AR. Cholesteryl ester transfer protein: A novel target for raising HDL and inhibiting atherosclerosis. Arterioscler Thromb Vasc Biol. 2003;23(2):160-167.

18. Niesor EJ. Different effects of compounds decreasing cholesteryl ester transfer protein activity on lipoprotein metabolism. Curr Opin Lipidol. 2011;22(4):288-295.

19. Arai T, Tsukada T, Murase T, Matsumoto K. Particle size analysis of high density lipoproteins in patients with genetic cholesteryl ester transfer protein deficiency. Clin Chim Acta. 2000;301(1-2):103-117.

20. Yamashita S, Sprecher DL, Sakai N, Matsuzawa Y, Tarui S, Hui DY. Accumulation of apolipoprotein E-rich high density lipoproteins in hyperalphalipoproteinemic human subjects with plasma cholesteryl ester transfer protein deficiency. J Clin Invest. 1990;86(3):688-695.

21. Ikewaki K, Nishiwaki M, Sakamoto T, et al. Increased catabolic rate of low density lipoproteins in humans with cholesteryl ester transfer protein deficiency. J Clin Invest. 1995;96(3):1573-1581.

22. Millar JS, Brousseau ME, Diffenderfer MR, et al. Effects of the cholesteryl ester transfer protein inhibitor torcetrapib on apolipoprotein B100 metabolism in humans. Arterioscler Thromb Vasc Biol. 2006;26(6):1350-1356.

23. Rosenson RS, Brewer HB Jr, Davidson WS, et al. Cholesterol efflux and atheroprotection: Advancing the concept of reverse cholesterol transport. Circulation. 2012;125(15):1905-1919.

24. Foger B, Chase M, Amar MJ, et al. Cholesteryl ester transfer protein corrects dysfunctional high density lipoproteins and reduces aortic atherosclerosis in lecithin cholesterol acyltransferase transgenic mice. J Biol Chem. 1999;274(52):36912-36920.

25. Hayek T, Masucci-Magoulas L, Jiang X, et al. Decreased early atherosclerotic lesions in hypertriglyceridemic mice expressing cholesteryl ester transfer protein transgene. J Clin Invest. 1995;96(4):2071-2074.
26. MacLean PS, Bower JF, Vadlamudi S, et al. Cholesteryl ester transfer protein expression prevents diet-induced atherosclerotic lesions in male $\mathrm{db} / \mathrm{db}$ mice. Arterioscler Thromb Vasc Biol. 2003;23(8):1412-1415.

27. Marotti KR, Castle CK, Boyle TP, Lin AH, Murray RW, Melchior GW. Severe atherosclerosis in transgenic mice expressing simian cholesteryl ester transfer protein. Nature. 1993;364(6432):73-75.

28. Plump AS, Masucci-Magoulas L, Bruce C, Bisgaier CL, Breslow JL, Tall AR. Increased atherosclerosis in ApoE and LDL receptor gene knockout mice as a result of human cholesteryl ester transfer protein transgene expression. Arterioscler Thromb Vasc Biol. 1999;19(4):1105-1110.

29. Westerterp M, van der Hoogt CC, de Haan W, et al. Cholesteryl ester transfer protein decreases high-density lipoprotein and severely aggravates atherosclerosis in APOE*3-leiden mice. Arterioscler Thromb Vasc Biol. 2006;26(11):2552-2559.

30. Tanigawa H, Billheimer JT, Tohyama J, Zhang Y, Rothblat G, Rader DJ. Expression of cholesteryl ester transfer protein in mice promotes macrophage reverse cholesterol transport. Circulation. 2007;116(11): $1267-1273$

31. Schwartz CC, VandenBroek JM, Cooper PS. Lipoprotein cholesteryl ester production, transfer, and output in vivo in humans. J Lipid Res. 2004;45(9):1594-1607.

32. Khera AV, Wolfe ML, Cannon CP, Qin J, Rader DJ. On-statin cholesteryl ester transfer protein mass and risk of recurrent coronary events (from the pravastatin or atorvastatin evaluation and infection therapythrombolysis in myocardial infarction 22 [PROVE IT-TIMI 22] study). Am J Cardiol. 2010;106(4):451-456.

33. Evans GF, Bensch WR, Apelgren LD, et al. Inhibition of cholesteryl ester transfer protein in normocholesterolemic and hypercholesterolemic hamsters: Effects on HDL subspecies, quantity, and apolipoprotein distribution. J Lipid Res. 1994;35(9):1634-1645.

34. Rittershaus CW, Miller DP, Thomas LJ, et al. Vaccine-induced antibodies inhibit CETP activity in vivo and reduce aortic lesions in a rabbit model of atherosclerosis. Arterioscler Thromb Vasc Biol. 2000;20(9):2106-2112.

35. Sugano M, Makino N, Sawada S, et al. Effect of antisense oligonucleotides against cholesteryl ester transfer protein on the development of atherosclerosis in cholesterol-fed rabbits. J Biol Chem. 1998;273(9): 5033-5036.

36. Matsuura F, Wang N, Chen W, Jiang XC, Tall AR. HDL from CETPdeficient subjects shows enhanced ability to promote cholesterol efflux from macrophages in an apoE- and ABCG1-dependent pathway. J Clin Invest. 2006;116(5):1435-1442.

37. Curb JD, Abbott RD, Rodriguez BL, et al. A prospective study of HDL-C and cholesteryl ester transfer protein gene mutations and the risk of coronary heart disease in the elderly. J Lipid Res. 2004;45(5): 948-953.

38. Moriyama Y, Okamura T, Inazu A, et al. A low prevalence of coronary heart disease among subjects with increased high-density lipoprotein cholesterol levels, including those with plasma cholesteryl ester transfer protein deficiency. Prev Med. 1998;27(5 Pt 1): 659-667.

39. Zhong S, Sharp DS, Grove JS, et al. Increased coronary heart disease in Japanese-American men with mutation in the cholesteryl ester transfer protein gene despite increased HDL levels. J Clin Invest. 1996;97(12): 2917-2923.

40. Thompson A, Di Angelantonio E, Sarwar N, et al. Association of cholesteryl ester transfer protein genotypes with CETP mass and activity, lipid levels, and coronary risk. JAMA. 2008;299(23):2777-2788.

41. Ridker PM, Pare G, Parker AN, Zee RY, Miletich JP, Chasman DI. Polymorphism in the CETP gene region, HDL cholesterol, and risk of future myocardial infarction: Genomewide analysis among 18 245 initially healthy women from the women's genome health study. Circ Cardiovasc Genet. 2009;2(1):26-33.

42. Voight BF, Peloso GM, Orho-Melander M, et al. Plasma HDL cholesterol and risk of myocardial infarction: A mendelian randomisation study. Lancet. 2012;380(9841):572-580.

43. Johannsen TH, Frikke-Schmidt R, Schou J, Nordestgaard BG, Tybjaerg-Hansen A. Genetic inhibition of CETP, ischemic vascular disease and mortality, and possible adverse effects. J Am Coll Cardiol. 2012;60(20):2041-2048. 
44. Clark RW, Ruggeri RB, Cunningham D, Bamberger MJ. Description of the torcetrapib series of cholesteryl ester transfer protein inhibitors, including mechanism of action. J Lipid Res. 2006;47(3):537-552.

45. Ranalletta M, Bierilo KK, Chen Y, et al. Biochemical characterization of cholesteryl ester transfer protein inhibitors. J Lipid Res. 2010;51(9): 2739-2752.

46. Brousseau ME, Schaefer EJ, Wolfe ML, et al. Effects of an inhibitor of cholesteryl ester transfer protein on HDL cholesterol. $N$ Engl $J$ Med. 2004;350(15):1505-1515.

47. Clark RW, Sutfin TA, Ruggeri RB, et al. Raising high-density lipoprotein in humans through inhibition of cholesteryl ester transfer protein: An initial multidose study of torcetrapib. Arterioscler Thromb Vasc Biol. 2004;24(3):490-497.

48. McKenney JM, Davidson MH, Shear CL, Revkin JH. Efficacy and safety of torcetrapib, a novel cholesteryl ester transfer protein inhibitor, in individuals with below-average high-density lipoprotein cholesterol levels on a background of atorvastatin. J Am Coll Cardiol. 2006;48(9): 1782-1790.

49. Morehouse LA, Sugarman ED, Bourassa PA, et al. Inhibition of CETP activity by torcetrapib reduces susceptibility to diet-induced atherosclerosis in New Zealand White rabbits. J Lipid Res. 2007;48(6): 1263-1272.

50. Barter PJ, Caulfield M, Eriksson M, et al. Effects of torcetrapib in patients at high risk for coronary events. $N$ Engl J Med. 2007;357(21): 2109-2122.

51. Kastelein JJ, van Leuven SI, Burgess L, et al. Effect of torcetrapib on carotid atherosclerosis in familial hypercholesterolemia. $N$ Engl J Med. 2007;356(16):1620-1630.

52. Bots ML, Visseren FL, Evans GW, et al. Torcetrapib and carotid intima-media thickness in mixed dyslipidaemia (RADIANCE 2 study): A randomised, double-blind trial. Lancet. 2007;370(9582):153-160.

53. Nissen SE, Tardif JC, Nicholls SJ, et al. Effect of torcetrapib on the progression of coronary atherosclerosis. N Engl J Med. 2007;356(13): 1304-1316.

54. Hu X, Dietz JD, Xia C, et al. Torcetrapib induces aldosterone and cortisol production by an intracellular calcium-mediated mechanism independently of cholesteryl ester transfer protein inhibition. Endocrinology. 2009;150(5):2211-2219.

55. Forrest MJ, Bloomfield D, Briscoe RJ, et al. Torcetrapib-induced blood pressure elevation is independent of CETP inhibition and is accompanied by increased circulating levels of aldosterone. Br J Pharmacol. 2008;154(7):1465-1473.

56. DePasquale M, Cadelina G, Knight D, et al. Mechanistic studies of blood pressure in rats treated with a series of cholesteryl ester transfer protein inhibitors. Drug Develop Res. 2009;70(10.1002/ddr.20282):35.

57. Clerc RG, Stauffer A, Weibel F, et al. Mechanisms underlying off-target effects of the cholesteryl ester transfer protein inhibitor torcetrapib involve L-type calcium channels. J Hypertens. 2010;28(8): 1676-1686.

58. Sofat R, Hingorani AD, Smeeth L, et al. Separating the mechanism-based and off-target actions of cholesteryl ester transfer protein inhibitors with CETP gene polymorphisms. Circulation. 2010;121(1): 52-62.

59. Blasi E, Bamberger M, Knight D, et al. Effects of CP-532,623 and torcetrapib, cholesteryl ester transfer protein inhibitors, on arterial blood pressure. J Cardiovasc Pharmacol. 2009;53(6):507-516.

60. Connelly MA, Parry TJ, Giardino EC, et al. Torcetrapib produces endothelial dysfunction independent of cholesteryl ester transfer protein inhibition. J Cardiovasc Pharmacol. 2010;55(5):459-468.

61. Simic B, Hermann M, Shaw SG, et al. Torcetrapib impairs endothelial function in hypertension. Eur Heart J. 2012;33(13):1615-1624.

62. Westerterp M, Koetsveld J, Tall AR. Cholesteryl ester transfer protein inhibition: A dysfunctional endothelium. J Cardiovasc Pharmacol. 2010;55(5):456-458.

63. Guerin M, Le Goff W, Duchene E, et al. Inhibition of CETP by torcetrapib attenuates the atherogenicity of postprandial TG-rich lipoproteins in type IIB hyperlipidemia. Arterioscler Thromb Vasc Biol. 2008;28(1): $148-154$.
64. Yvan-Charvet L, Matsuura F, Wang N, et al. Inhibition of cholesteryl ester transfer protein by torcetrapib modestly increases macrophage cholesterol efflux to HDL. Arterioscler Thromb Vasc Biol. 2007;27(5): 1132-1138.

65. Tall AR. The effects of cholesterol ester transfer protein inhibition on cholesterol efflux. Am J Cardiol. 2009;104(Suppl 10):39E-45E.

66. Tchoua U, D'Souza W, Mukhamedova N, et al. The effect of cholesteryl ester transfer protein overexpression and inhibition on reverse cholesterol transport. Cardiovasc Res. 2008;77(4):732-739.

67. Briand F, Thieblemont Q, Andre A, Ouguerram K, Sulpice T. CETP inhibitor torcetrapib promotes reverse cholesterol transport in obese insulin-resistant CETP-ApoB100 transgenic mice. Clin Transl Sci. 2011;4(6):414-420.

68. Nicholls SJ, Tuzcu EM, Brennan DM, Tardif JC, Nissen SE. Cholesteryl ester transfer protein inhibition, high-density lipoprotein raising, and progression of coronary atherosclerosis: Insights from ILLUSTRATE (investigation of lipid level management using coronary ultrasound to assess reduction of atherosclerosis by CETP inhibition and HDL elevation). Circulation. 2008;118(24):2506-2514.

69. Fryirs MA, Barter PJ, Appavoo M, et al. Effects of high-density lipoproteins on pancreatic beta-cell insulin secretion. Arterioscler Thromb Vasc Biol. 2010;30(8):1642-1648.

70. Barter PJ, Rye KA, Tardif JC, et al. Effect of torcetrapib on glucose, insulin, and hemoglobin A1c in subjects in the investigation of lipid level management to understand its impact in atherosclerotic events (ILLUMINATE) trial. Circulation. 2011;124(5):555-562.

71. Funder JW. Aldosterone, hypertension and heart failure: Insights from clinical trials. Hypertens Res. 2010;33(9):872-875.

72. Kuivenhoven JA, de Grooth GJ, Kawamura H, et al. Effectiveness of inhibition of cholesteryl ester transfer protein by JTT-705 in combination with pravastatin in type II dyslipidemia. Am J Cardiol. 2005;95(9): $1085-1088$

73. Okamoto H, Yonemori F, Wakitani K, Minowa T, Maeda K, Shinkai H. A cholesteryl ester transfer protein inhibitor attenuates atherosclerosis in rabbits. Nature. 2000;406(6792):203-207.

74. de Grooth GJ, Kuivenhoven JA, Stalenhoef AF, et al. Efficacy and safety of a novel cholesteryl ester transfer protein inhibitor, JTT-705, in humans: A randomized phase II dose-response study. Circulation. 2002;105(18):2159-2165

75. Niesor EJ, Magg C, Ogawa N, et al. Modulating cholesteryl ester transfer protein activity maintains efficient pre-beta-HDL formation and increases reverse cholesterol transport. J Lipid Res. 2010;51(12): 3443-3454.

76. Derks M, Anzures-Cabrera J, Turnbull L, Phelan M. Safety, tolerability and pharmacokinetics of dalcetrapib following single and multiple ascending doses in healthy subjects: A randomized, double-blind, placebo-controlled, phase I study. Clin Drug Investig. 2011;31(5): 325-335.

77. Stein EA, Roth EM, Rhyne JM, Burgess T, Kallend D, Robinson JG. Safety and tolerability of dalcetrapib (RO4607381/JTT-705): Results from a 48-week trial. Eur Heart J. 2010;31(4):480-488.

78. Fayad ZA, Mani V, Woodward M, et al. Safety and efficacy of dalcetrapib on atherosclerotic disease using novel non-invasive multimodality imaging (dal-PLAQUE): A randomised clinical trial. Lancet. 2011;378(9802):1547-1559.

79. Luscher TF. Effects of dalcetrapib on vascular function: Results of phase IIb dal-VESSEL study. Available from: http://www.escardio. org/about/press/press-releases/esc11-paris/Pages/HL1-dal-VESSEL. aspx. Updated 2011. Accessed February 1, 2012.

80. Schwartz GG, Olsson AG, Ballantyne CM, et al. Rationale and design of the dal-OUTCOMES trial: Efficacy and safety of dalcetrapib in patients with recent acute coronary syndrome. Am Heart J. 2009;158(6): 896-901. e3.

81. Miller R. Roche stops dalcetrapib trial for lack of benefit. Available from: http://www.theheart.org/article/1395141.do. Updated 2012. Accessed June 21, 2012

82. Schwartz GG, Olsson AG, Abt M, et al. Effects of dalcetrapib in patients with a recent acute coronary syndrome. $N$ Engl $\mathrm{J} \mathrm{Med.}$ 2012;367(22):2089-2099 
83. Nicholls SJ, Brewer HB, Kastelein JJ et al. Effects of the CETP Inhibitor evacetrapib administered as monotherpay or in combination with statins on HDL and LDL cholesterol: a randomized controlled trial. JAMA. 2011;306(19):2099-2109.

84. Eli Lilly and Company. A study of evacetrapib in high-risk vascular disease (ACCELERATE); NCT01687998. Available from: http:// www.clinicaltrials.gov/ct2/show/study/NCT01687998?term=eva cetrapib\&rank=3. Updated 2012. Accessed November 17, 2012.

85. Smith CJ, Ali A, Hammond ML, et al. Biphenyl-substituted oxazolidinones as cholesteryl ester transfer protein inhibitors: Modifications of the oxazolidinone ring leading to the discovery of anacetrapib. J Med Chem. 2011;54(13):4880-4895.

86. Gutstein DE, Krishna R, Johns D, et al. Anacetrapib, a novel CETP inhibitor: Pursuing a new approach to cardiovascular risk reduction. Clin Pharmacol Ther. 2012;91(1):109-122.

87. Kumar S, Tan EY, Hartmann G, et al. Metabolism and excretion of anacetrapib, a novel inhibitor of the cholesteryl ester transfer protein, in humans. Drug Metab Dispos. 2010;38(3):474-483.

88. Dansky HM, Bloomfield D, Gibbons P, et al. Efficacy and safety after cessation of treatment with the cholesteryl ester transfer protein inhibitor anacetrapib (MK-0859) in patients with primary hypercholesterolemia or mixed hyperlipidemia. Am Heart J. 2011;162(4): 708-716.

89. Bloomfield D, Carlson GL, Sapre A, et al. Efficacy and safety of the cholesteryl ester transfer protein inhibitor anacetrapib as monotherapy and coadministered with atorvastatin in dyslipidemic patients. Am Heart J. 2009;157(2):352-360. e2.

90. Krishna R, Anderson MS, Bergman AJ, et al. Effect of the cholesteryl ester transfer protein inhibitor, anacetrapib, on lipoproteins in patients with dyslipidaemia and on 24-h ambulatory blood pressure in healthy individuals: Two double-blind, randomised placebo-controlled phase I studies. Lancet. 2007;370(9603):1907-1914.

91. Krauss RM, Wojnooski K, Orr J, et al. Changes in lipoprotein subfraction concentration and composition in healthy individuals treated with the CETP inhibitor anacetrapib. J Lipid Res. 2012;53(3): 540-547.
92. Krishna R, Bergman AJ, Green M, Dockendorf MF, Wagner JA, Dykstra K. Model-based development of anacetrapib, a novel cholesteryl ester transfer protein inhibitor. AAPS J. 2011;13(2): 179-190.

93. Yvan-Charvet L, Kling J, Pagler T, et al. Cholesterol efflux potential and antiinflammatory properties of high-density lipoprotein after treatment with niacin or anacetrapib. Arterioscler Thromb Vasc Biol. 2010;30(7):1430-1438.

94. Castro-Perez J, Briand F, Gagen K, et al. Anacetrapib promotes reverse cholesterol transport and bulk cholesterol excretion in Syrian golden hamsters. J Lipid Res. 2011;52(11):1965-1973.

95. Cannon CP, Dansky HM, Davidson M, et al. Design of the DEFINE trial: Determining the EFficacy and tolerability of CETP INhibition with AnacEtrapib. Am Heart J. 2009;158(4):513-519. e3.

96. Cannon CP, Shah S, Dansky HM, et al. Safety of anacetrapib in patients with or at high risk for coronary heart disease. $N$ Engl J Med. 2010;363(25):2406-2415.

97. Davidson M, Liu SX, Barter P, et al. Measurement of LDL-C after treatment with the CETP inhibitor anacetrapib. J Lipid Res. 2013;54(2):467-472.

98. Brinton E, Liu S, Stepanavage M, et al. Lipid-modifying effects of anacetrapib in patients with lower versus higher baseline levels of HDL-C, LDL-C, and TG: Pre-specified subgroup analyses of the DEFINE (determining the efficacy and tolerability of CETP INhibition with AnacEtrapib) trial. Circulation. 2011;124:A9649.

99. Gotto A, Cannon C, Shah S, et al. Lipid modifying effects of anacetrapib: Pre-specified subgroup analyses. Circulation. 2011;124: A15035.

100. Bowman L. REVEAL: Randomized EValuation of the effects of anacetrapib through lipid-modification. Available from: http://www. clinicaltrials.gov/ct2/show/NCT01252953?term=anacetrapib\&rank=4. Updated 2011. Accessed June 25, 2012.

101. Cannon CP. High-density lipoprotein cholesterol as the Holy Grail. JAMA. 2011;306(19):2153-2155
Research Reports in Clinical Cardiology

\section{Publish your work in this journal}

Research Reports in Clinical Cardiology is an international, peerreviewed, open access journal publishing original research, reports, editorials, reviews and commentaries on all areas of cardiology in the clinic and laboratory. The manuscript management system is completely online and includes a very quick and fair peer-review system.

\section{Dovepress}

Visit http://www.dovepress.com/testimonials.php to read real quotes from published authors. 